References:

1 Seredyuk M., Muñoz M.C., Castro M., Romero-Morcillo T., Gaspar A.B. \& Real J.A.(2013). Chemistry - A European Journal. $196591-6596$

Keywords: Photoswitcable materials, Spin Crossover, structure
MS36-P27

\section{Halogen bonding, actinide contraction and coordination modes of ligands in uranyl, neptunyl and plutonyl trichloroacetates with ammonium cations}

Anton Savchenkov ${ }^{1}$, Andrei Uhanov ${ }^{1}$, Mikhail Grigoriev ${ }^{2}$, Aleksandr Fedoseev $^{2}$, Denis Pushkin ${ }^{1}$, Larisa Serezhkina ${ }^{1}$, Viktor Serezhkin ${ }^{1}$

1. Samara National Research University, Samara, Russia

2. Russian academy of sciences A.N. Frumkin Institute of Physical Chemistry and Electrochemistry, Moscow, Russia

email: anton.savchenkov@gmail.com

Uranyl, neptunyl and plutonyl ions usually form series of isostructural compounds. This fact is very favorable for investigation of the relationship between composition, structure and properties, since it is possible to obtain series of compounds with single varying parameter, for example, the actinide atom, the ligand or the counterion.

In order to obtain new series of $\mathrm{U}, \mathrm{Np}, \mathrm{Pu}$ compounds, we attempted to use trichloroacetate ions as ligands. As a result, single crystals of isostructural compounds $\left(\mathrm{NH}_{4}\right)_{4} \mathrm{AnO}_{2}(\mathrm{t}-$ ca $)_{6}(\mathrm{Htca})\left(\mathrm{H}_{2} \mathrm{O}\right)_{3}$ (where $\mathrm{An}$ is $\mathrm{U}, \mathrm{Np}$ or $\mathrm{Pu}$ and tca is a trichloroacetate ion) were grown and studied using X-ray diffraction analysis. These compounds are of great importance for studying actinide contraction as well as halogen bonding. For example, it has recently been shown that bonds involving halogen atoms can serve as synthons in the formation of supramolecular architectures as well as chemical bonds in the presence of secondary metal atoms in uranyl carboxylate complexes [1]. Halogen bonds in the title crystal structures were analyzed using the method of molecular Voronoi-Dirichlet polyhedra. Actinide contraction in the row of hexavalent $\mathrm{U}, \mathrm{Np}$ and $\mathrm{Pu}$ atoms is discussed on the example of all available series of isostructural compounds.

Aspects of theoretical crystal chemistry are also discussed in the current project. With an increase in the number of structurally studied coordination compounds, it becomes necessary to systematize them in order to facilitate the search for analogous structures, their comparison and identification of common trends in coordination chemistry. One of the methods of description and systematization of complex compounds is the use of crystal-chemical formulae (CCF) [2]. With the help of CCF it is possible to describe any mono- and polynuclear, as well as homo- and heteroligand complexes. CCF's are concise and can be recorded and used automatically by computer algorithms. However, the discussion of halogen-substituted organic ligands in the title compounds required an additional improvement of the recording of $\mathrm{CCF}$ and of coordination modes of ligands. In the course of this project, we analyzed coordination modes of trichloroacetate ions in all compounds from CSD [3], what is of great importance for crystal structure prediction and crystal design.

This research was supported by the Russian Science Foundation (project №17-73-10117).

\section{References:}

[1] Kalaj, M. et al. (2017). Inorg. Chem. 56, 9156-9168.

[2] Serezhkin, V. N. et al. (2009). Acta Cryst. B65, 45-53.

[3] Groom, C. R. et al. (2016). Acta Cryst. B72, 171-179. 